\title{
EL PROYECTO EDUCATIVO COMO ELEMENTO VERTEBRADOR DE LA PRÁCTICA PEDAGÓGICA: REFLEXIONES Y PROPUESTAS PARA LA ACCIÓN
}

\author{
The Educational Project as a pivotal framework for \\ pedagogical practice: reflections and proposals for action
}

\section{Le Projet Éducatif comme axe de soutien de la pratique pédagogique: réflexions et approches pour l'action}

Javier Argos GonzÁlez, María Pilar Ezquerra Muñoz y Ana Castro Zubizarreta Universidad de Cantabria. Facultad de Educación. Departamento de Educación. Edificio Interfacultativo. Avda. Los Castros, s/n. 39005 Santander (Cantabria). Correo-e: argosj@unican.es; ezquermp@unican.es; castroza@unican.es

Fecha de recepción: marzo de 2010

Fecha de aceptación definitiva: julio de 2010

Biblid [(1130-3743) 22, 2-2010, 183-205]

RESUMEN

La tarea educativa presenta un cariz lo suficientemente complejo y relevante como para demandar su "construcción" desde marcos teórico-pedagógicos consistentes, rigurosos y éticamente fundamentados. Para conseguir este propósito, la coherencia y el sentido de dicha tarea se convierten en elementos imprescindibles que se han de proyectar tanto a nivel de centro educativo como a nivel de aula que es, en última instancia, donde se concretan las grandes líneas pedagógicas asumidas consensuadamente.

Teniendo en cuenta estas consideraciones, en el presente trabajo analizamos la importancia del Proyecto Educativo de Centro como texto sustentador de la tarea 
pedagógica, para plantear a continuación un conjunto de postulados que se deberían contemplar en nuestra práctica profesional. Así, elementos como la pedagogía de la escucha, el aprendizaje lento, y la intuición, la creatividad, la curiosidad y el placer por aprender emergen y se visibilizan en la escena pedagógica.

Palabras clave: Proyecto Educativo de Centro, dimensión ética, relación educativa, principios pedagógicos

\section{SUMMARY}

The basic features of an educational task are sufficiently complex to demand their "construction" from theoretical-pedagogical frameworks that are consistent, rigorous and ethically founded. To achieve this end, the coherence and meaning of such a task become transformed into indispensable elements which need to be projected, both into the school and into the classroom, which, in the last resort, is where the important pedagogical formulae that have been adopted on consensus are given shape.

Taking these considerations into account, we analyse in the present research the importance of the Educational School Project, as a text which upholds the pedagogical task, before going on to formulate a series of postulates which should be upheld in our professional practice. Thus elements such as the pedagogy of listening, unhurried learning, and intuition, creativity, curiosity and pleasure in learning emerge and are visible in the pedagogical scene.

Key words: Educational School Project, ethical dimension, educational relationship, pedagogical principles.

\section{SOMMAIRE}

La tâche éducative par son importance et sa complexité demande un travail de "Construction" qui intègre des cadres théoriques et pédagogiques solides, rigoureux et éthiquement fondés. Pour en arriver là, la cohérence et le sens de cette tâche deviennent des éléments essentiels qui doivent se projeter aussi bien au niveau du centre éducatif comme au niveau de la classe, qui est, en dernier recours, le lieu où se concrétisent les grandes lignes pédagogiques assumées de manière consensuelle.

En tenant compte de ces considérations, nous analyserons dans l'étude suivante l'importance du Projet Éducatif du centre scolaire comme texte, support de la tâche pédagogique, pour exposer ensuite un ensemble de postulats qui devraient être pris en compte dans notre pratique professionnelle. Ainsi, des éléments comme la pédagogique de l'écoute, l'apprentissage lent, l'intuition, la créativité, la curiosité et le plaisir d'apprendre, émergent et se font présents sur la scène pédagogique.

Mots clés: Projet Éducatif du Centre scolaire, dimension éthique, relation éducative, principes pédagogiques. 
Como es bien sabido, la complejidad de la tarea pedagógica es algo intrínseco al hecho relacional e intersubjetivo que comporta. Y la resolución satisfactoria de esa tarea, no sólo como algo objetivable externamente, sino, fundamentalmente, como realidad y percepción subjetiva de los diferentes actores implicados en el escenario pedagógico escolar, pasa, a nuestro entender, por tomar en consideración preferente la coherencia y el sentido de la práctica profesional ya que éstos se convierten en importantes axiomas y postulados reguladores de la calidad que en todo momento hemos de perseguir en nuestro quehacer educativo.

Para conseguir este propósito, de forma valiosa y comprometida, sería necesario considerar tanto los referentes y principios pedagógicos consensuados por una determinada comunidad educativa y contemplados en su Proyecto Educativo, como la perspectiva proyectada por cada educador en forma de una serie de vectores de acción en el desarrollo de su actividad cotidiana.

Plantearemos, a lo largo de nuestra exposición, un conjunto de constataciones y reflexiones desde las que argumentamos la necesidad de contar con referentes valiosos en nuestra tarea pedagógica, que se sustentan en algunas miradas y se nutren de ciertos matices escasamente considerados en los escenarios escolares. En relación con ellas, apuntamos algunas pautas orientativas que puedan servir de referencia para el desarrollo de la práctica educativa.

\section{El Proyecto Educativo de Centro como texto sustentador de nuestra tarea PEDAGÓGICA}

La preocupación suscitada en el ámbito educativo por los proyectos educativos de los centros escolares no es algo nuevo. Así, tanto desde las administraciones educativas como desde los equipos directivos de los centros y de los propios docentes, se han podido ir constatando demandas y respuestas de diferente tipología, alcance y nivel de concreción.

Creemos que el potencial pedagógico que encierra el Proyecto Educativo de Centro es algo innegable aunque, como acertadamente apuntan Beltrán y San Martín (2000), la mera incorporación del término al argot escolar no presupone la transformación significativa de las prácticas educativas reales. Es por ello por lo que entendemos con ellos que merece la pena retomar el concepto y buscarle nuevos significados, tratando de aprovechar toda la capacidad que potencialmente tiene para introducir nuevas pautas de acción que dinamicen la tarea educativa en el ámbito escolar.

El PEC aparece contemplado en el artículo 121 de la LOE, dentro del Capítulo ir relativo a la Autonomía de los centros y ubicado, a su vez, dentro del Título v concerniente a la Participación, autonomía y gobierno de los centros.

De este marco legislativo se desprende una tarea inexcusable y prioritaria a emprender por parte de las comunidades educativas escolares: la construcción o, si se quiere, la "reconstrucción" de ese Proyecto en clave profunda y fundamentada y 
no desde el mero formalismo que se limita a dar respuesta a las demandas externas efectuadas por la Administración Educativa.

Para intentar conseguir este propósito deberíamos tomar en consideración algunos referentes o axiomas que, ubicados en diferentes ámbitos, podrían, a nuestro entender, ayudarnos a huir de formulaciones y planteamientos estereotipados y burocráticos a la hora de "construir" nuestro Proyecto.

El primero de esos ámbitos tiene que ver con el sentido que, en todo momento, ha de poseer el texto, el Proyecto. Y, para que esto ocurra, entendemos que es inexcusable la referencia que él ha de hacer a los sustratos axiológicos y teleológicos en cuanto que elementos determinantes de la orientación o perspectiva (concretada en valores y metas) que considere la comunidad educativa como prioritaria en la tarea educativa. Utilizando un lenguaje metafórico, podríamos expresarlo como el lado oculto de la luna que, aunque no siempre se haga explícito, existe y contribuye a dotar de sentido integral al quehacer pedagógico cotidiano.

Otro ámbito haría referencia al contenido del texto. Dentro de éste, podríamos apuntar una serie de referentes importantes como son los relativos a la relevancia de los elementos que lo integren (con unos pocos mimbres se puede hacer un buen texto), entendiendo que no se trata tanto de contemplar un amplio listado de referentes orientadores de la praxis pedagógica sino, más bien, un conjunto limitado de elementos potentes que otorguen significado a ésta. También sería fundamental que se produjera una cierta inclusividad entre los diferentes proyectos que están integrados en el Proyecto global (juntos pero no revueltos) y a los que posteriormente aludiremos. Por último, el contenido del Proyecto debería permitirnos otorgar coherencia (no sólo hablar sino, también, predicar con el ejemplo) a lo proyectado y, como obligada consecuencia ética, también a su concreción en el marco del proceso educativo.

El tercero y último ámbito referencial o axiomático estaría relacionado con la dinámica del texto, esto es, con todo lo relacionado con su génesis, su desarrollo y su valoración. En relación con ello podríamos apuntar como ingredientes importantes el realismo que ha de tener, aunque no sea ajeno al apunte de ciertas metas pedagógicas mediatas (el tocar tierra de Sancho con algunas dosis de la utopía del Hidalgo), la dialéctica intersubjetiva necesaria para generar propuestas y planteamientos de "segundo orden" (cuatro ojos ven más que dos) y la reformulación continua que, en definitiva, es la concreción de su dinamismo intrínseco (el movimiento se demuestra andando) y el de las características propias de la realidad pedagógica a la que hace referencia.

Desde las anteriores consideraciones planteamos lo que, a nuestro entender, debería de ser el PEC, entendiéndolo como un texto situado, es decir, ubicado en un determinado contexto; como un texto que dé sentido, coherencia, realismo y dinámica a la tarea pedagógica.

En relación con ello, estamos totalmente de acuerdo con Puig (2000) al entender que resulta imposible lograr una escuela democrática sin contar con valores 
que orienten su funcionamiento, esto es, con valores que, en definitiva, dinamicen la acción educativa al contar con el aprecio de la comunidad escolar.

Ahora bien, no hace falta efectuar una observación excesivamente escrupulosa para constatar la presencia de diferentes niveles de exigencia y de implicación personal e institucional en el proceso de construcción y desarrollo de un Proyecto como el que nos ocupa. Algunos de ellos, apuntados sintéticamente, serían los siguientes:

\section{- Nivel de implicación escasa o prácticamente nula}

Desde nuestra perspectiva, los principales motivos para que esta carencia de implicación se produzca son, fundamentalmente, tres. El primero sería el desconocimiento del valor potencial que posee el PEC, como posteriormente apuntaremos; el segundo la desmotivación ante la tarea de construir el texto, debida a inercias personales y/o institucionales (tanto las objetivamente constatadas como las percibidas como tales), y, finalmente, otro motivo estaría vinculado con la asimilación de la tarea de construcción que hemos de emprender a esquemas y/o a experiencias previas poco satisfactorias.

\section{- Nivel de implicación limitada y escasamente productiva}

Entre las causas que percibimos como generadoras dentro de este nivel de implicación y exigencia podríamos apuntar las siguientes: la primera, y muy importante, sería la balcanización del trabajo producida como consecuencia de aportaciones yuxtapuestas, no integradas en la cultura y el quehacer educativo del centro, por parte de diferentes actores implicados en ese escenario educativo; la segunda causa guardaría relación con las miradas superficiales o epidérmicas efectuadas a los procesos y tareas a asumir por parte de ellos; por último, constataríamos como tercer motivo de este nivel de implicación, la disyunción o fractura existente entre el Proyecto pedagógico entendido como construcción teórica y su encarnación en la praxis educativa de la institución escolar.

\section{- Nivel de implicación elevada}

A diferencia de los anteriores niveles, éste se genera en torno a tres grandes ejes que se constatan cuando efectuamos un estudio profundo y cercano, antropológico diríamos, a una determinada comunidad educativa: el primero sería el sentido encontrado al Proyecto, no ya sólo como producto -que también- sino, fundamentalmente, como proceso continuo e inacabado; en segundo lugar aparecería como referente el compromiso asumido por todos, derivado de la comprensión del sentido más amplio y profundo del PEC; y, por último, como otro necesario ingrediente, una cierta dosis de utopía para no limitarnos sólo a llevar a cabo la tarea educativa, sino también para idearla y, por qué no, también para soñarla. 
De lo sintéticamente apuntado hasta ahora se desprende, para nosotros, una constatación importante: la imposibilidad de construir el texto educativo que debe de constituir el Proyecto en el vacío.

Este vacío debe evitarse a toda costa, dado que desempeña siempre un papel dañino para la construcción de cualquier Proyecto fundamentado y comprometido. En caso de darse ese vacío, se constata y encarna, dependiendo de cada centro educativo, en diferentes ámbitos y tipos. Así, al menos potencialmente, podríamos contemplar los que denominamos contextual, de modelo y de consensos.

El vacío contextual sería aquel que se produce cuando no analizamos nuestro entorno escolar de la forma profunda y detenida que debiéramos. Por su parte, el vacío de modelo se hace visible cuando no contemplamos y tomamos en consideración una serie de referentes filosófico-pedagógicos imprescindibles en la construcción de cualquier modelo pedagógico. Por último, el vacío de consensos se manifestaría como consecuencia "lógica" de no mirar al otro, a los otros, con la pretensión de crear con ellos lo que Assmann (2002) denomina interfases entre sus campos de sentido.

Sólo desde la consideración y superación de cualquiera de los vacíos apuntados, estaríamos en condiciones de dotar de sentido y valor al Proyecto que entre todos queremos construir y que se convierte en elemento imprescindible para una tarea educativa profunda, sólida y fundamentada.

Entre las orientaciones o pautas que se pueden inferir de lo anterior planteado de cara al desarrollo de la acción pedagógica, indicaríamos las siguientes:

- Es preciso alcanzar consensos por parte de las comunidades educativas de los centros -fundamentalmente por los docentes- acerca de los valores y metas orientadores de su tarea pedagógica. La exigencia derivada de ello no tendría que ubicarse sólo en un plano declarativo sino que debería de comportar una explicitación de los contextos y modos en los que aquéllos se concretan en la cotidianeidad educativa, tanto a nivel de centro como de aula.

A lo largo de nuestra actividad formativa y de asesoría a centros, hemos podido constatar, con mayor frecuencia de lo que sería deseable, tanto ausencia y/o desconocimiento de los referidos consensos, como una indefinición de las "proyecciones" o concreciones del contenido de los mismos en la realidad pedagógica cotidiana.

- Otra tarea importante a acometer sería la relacionada con el obligado proceso de "filtrado" o de "depuración selectiva" respecto de aquel puñado de referentes que, a modo de grandes orientaciones filosófico-pedagógicas, hacen que tenga sentido la actuación docente y la de la vida educativa de los centros.

Nuestra experiencia en relación con este proceso revela cómo esta tarea requiere de unas altas dosis de reflexión y de análisis pedagógico profundos. El "optar" por ciertos valores y metas supone también "renunciar" a 
otros o, en ocasiones, integrarlos en los concebidos como prioritarios. Limitarse a "listar" valores y propósitos educativos suele generar vaguedad de planteamientos y escasas dosis de compromiso profesional compartido.

\subsection{El sentido, las funciones y el valor del texto}

No es excesivamente difícil constatar, tanto en comunidades educativas consideradas en su conjunto, como en el caso de docentes concretos, el escaso reconocimiento que otorgan al texto en cuanto que referente insustituible de la praxis pedagógica.

Desde esta constatación, y aunque sólo sean apuntados de forma telegráfica, planteamos una serie de elementos argumentativos que, complementándose, ponen en evidencia la relevancia que el Proyecto Educativo de Centro comporta en el desarrollo profundo y comprometido de toda comunidad educativo-escolar. Entre los principales elementos argumentales constataríamos los siguientes:

\section{- El texto como pretexto para el encuentro}

El tiempo compartido y la tarea proyectada con el otro, con los otros, se convierte en una herramienta valiosísima para el "obligado" encuentro, a la vez que en potencial generador de posteriores encuentros enriquecedores con diferentes personas e instancias.

\section{- El texto como encuentro de subjetividades}

Como acertadamente afirman Illeras y Medina (2006), si pretendemos construir una escuela radicalmente democrática, es necesario partir de teorías y prácticas que constaten que todas las personas tenemos capacidades. Porque, como apuntan Bárcena y Mélich (2000), el aprender sólo es posible en el marco de relaciones entre diversas subjetividades.

En definitiva, el Proyecto pedagógico del centro reclamaría de todos los actores vinculados con el escenario escolar un proceso de concienciación sobre el valor de lo subjetivo en cuanto referente pedagógico fundamental y, también, sobre lo intersubjetivo entendido como contexto para un verdadero encuentro que comportaría actitudes y conductas de escucha, diálogo y respeto entre todas las personas implicadas. Supondría, en definitiva, intentar alcanzar el propósito que Esteban y Buxarrais (2004) plantean en el ámbito universitario: permitir a cada educador escribir narraciones personales de vida insertas en un proyecto común.

\section{- El texto como conjunto de acuerdos formalizados}

La construcción del Proyecto supondría emprender un trabajo educativo compartido que se concretaría en un marco de acción común y no en la confección de 
un simple documento a elaborar desde demandas y prescripciones recibidas por parte de la Administración Educativa.

Como apunta Bolívar (1998), habría que ir más allá de la mera planificación racional conforme a las referidas demandas. Ello supondría invertir tiempos y energías que nos aportan poco, más aún pensando -como él lo hace- que los valores y las finalidades no preexisten en la acción sino que se generan en el curso mismo de las tareas y la vida de un centro.

\section{- El texto como crisol de otros textos}

Nos parece imprescindible la necesidad, por parte de la escuela, de contar con un "instrumento simbólico que sea capaz de representar de manera armonizada los distintos intereses que se ponen de manifiesto en su día a día" (Beltrán y San Martín, 2000), así como -añadiríamos nosotros- para integrar y dotar de direccionalidad, coherencia y sentido a todos los proyectos y planes desarrollados en el centro.

En esta línea, un riesgo a evitar que, por desgracia, aparece con excesiva frecuencia en nuestros contextos escolares, sería el de la disgregación, difuminación y/o yuxtaposición de ese conjunto de proyectos y planes dentro del PEC (Plan de Convivencia, Plan de Acción Tutorial, Plan de Interculturalidad...). Cuando esto ocurre, esto es, cuando no existe una verdadera integración entre ellos dentro del todo que debe de conformar el texto, su potencialidad disminuye, perdiendo el Proyecto coherencia y perspectiva.

\section{- El texto como referente ético y compromiso de la praxis pedagógica}

La dimensión ética a la que, de forma explícita o implícita, nos debe remitir el PEC se conforma, a nuestro entender, como una de sus funciones nucleares. Así, como apunta Gather Thurler (2004), la elaboración de cualquier proyecto de una institución educativa se convierte en un objetivo simbólico, orientado por valores, visiones políticas y sociales, como una ética, no teniendo sentido entenderlo como un mero programa operativo, como una programación que debe obligadamente dar respuesta al fin previsto conforme a una secuencia predeterminada.

Cambiar y mejorar los entornos educativos implica siempre, según Angulo (2002), un propósito moral. Los cambios se sustentan en propósitos morales de mejora, esto es, en valores, formas de convivencia y elecciones de los colectivos. Ignorar este sustrato supondría para este autor intentar ocultar la raíz misma de la acción humana. El Proyecto Educativo se convierte en el texto institucional más adecuado para dejar constancia de nuestro compromiso ante todo ello y nos obliga a tratarlo como un documento altamente significativo como referente claro de valor (Puig y Martín, 1998). En definitiva, contribuiría, utilizando la expresión de García Gómez (2006), a facilitar referencias éticas de identidad. 


\section{- El texto como referente útil para valorar logros y expectativas}

Por último, entendemos que los contenidos contemplados en el PEC deben convertirse en el principal referente para evaluar los logros que vamos alcanzando en el seno de la institución escolar.

Para nosotros, este referente es fundamental en la medida en que se apoya en lo que algunos autores denominan consensos situados, esto es, pegados a nuestro contexto, y no tanto a consensos macro sobre educación desvinculados o, con una referencia más que remota, al escenario educativo concreto. Es por ello por lo que, inexcusablemente, nos encontramos con el compromiso, tanto epistemológico como profesional, de relacionar texto y contexto.

Los anteriores apuntes acerca del sentido, funciones y valor del texto que es el PEC nos llevan a efectuar una serie de consideraciones y de propuestas para la acción. Éstas son las siguientes:

- Se convierte en una tarea inexcusable para las comunidades educativas de los centros el encontrar y explicitar tiempos para el encuentro entre personas, fundamentalmente docentes, que permitan crear "espacios compartidos" y alcanzar acuerdos no sólo avalados desde el formalismo sino desde el convencimiento de lo que perseguimos en nuestra tarea educativa.

Son frecuentes los casos en los que ciertas actitudes prejuiciosas hacia otros colegas o miembros de la comunidad educativa del centro se han disipado mediante la convivencia producto de esos marcos de encuentro. Ello, obviamente, requiere, como paso previo, el determinar momentos en el calendario y horario escolares que eviten el riesgo de "dejar para un mejor momento" el compartir planteamientos, experiencias y propuestas para la mejora de la práctica pedagógica. Y, también, comporta el compromiso por todos los implicados de una participación activa en las sesiones y en las tareas derivadas de ellas.

- Otro reto importante y dificultoso a plantearse es el vinculado con la integración de los diferentes proyectos que actualmente han de llevarse a cabo en los centros y que están integrados en el PEC (Plan de Convivencia, Plan de Acción Tutorial, Plan de Interculturalidad...).

Este reto se convierte en obligado aunque sólo sea por evitar la percepción que tienen muchos centros de estar "abrumados" por los requerimientos derivados de tantos planes institucionales. A nuestro entender, la disipación o la mitigación de esta situación pasa por encontrar "bisagras" potentes que los articulen y cohesionen, siendo el PEC y sus principales referentes axiológicos y teleológicos quienes, creemos, pueden mejor desempeñar esa función, tanto en el diseño de los planes y proyectos como en su desarrollo y en la evaluación de los logros alcanzados y/o pretendidos. 


\subsection{El Proyecto Educativo de Centro (el texto) en un contexto}

Aun siendo los argumentos anteriores elementos fundamentales en la construcción del texto que conforma el PEc, reiteramos nuestra profunda convicción de que éste no tendría ningún valor o potencialidad pedagógica si no se construye en consonancia y tomando como referente prioritario el marco peculiar en el que se inscribe la acción educativa de un determinado centro.

A pesar de que este axioma, al menos en apariencia, parece considerarse y ser tenido en cuenta a la hora de formular todo proyecto, a poco que observemos con detenimiento su proceso de construcción, nos encontramos con diferentes miradas o formas de entender ese contexto. Apuntamos a continuación tres de ellas que hemos tenido ocasión de constatar.

\section{- El contexto visto o la mirada epidérmica}

Ubicarnos en esa primera mirada al contexto supondría limitarnos a mirar en clave superficial los diferentes elementos conformadores de un determinado contexto educativo-escolar.

Algunos ejemplos de actitudes y conductas limitadoras de este tipo se constatarían en afirmaciones o expresiones reveladoras del empobrecido nivel de análisis en el que nos situamos. Así, por ejemplo, utilizar la expresión "profesorado envejecido" entendida desde la superficialidad valorativa de atender sólo a la edad cronológica de los docentes, ignorando otra serie de elementos con mayor potencia explicativa como, por ejemplo, su experiencia o su implicación personal e institucional. O, también, como otra muestra del mismo fenómeno, encontrar alusiones a "familias poco estimulantes a nivel educativo", reveladora de una mirada al contexto familiar sólo en clave estático-estructural -fisonomía familiar- y no en clave dinámico-funcional y educativa.

\section{- El contexto percibido o la mirada pensada}

Esta segunda forma de mirar el contexto de la institución educativa supondría una aproximación más sólida y profunda. Así, comportaría analizar reflexiva y racionalmente el entorno o, mejor diríamos, los entornos, en los que se inscribe el centro escolar, incluido el microentorno que él mismo conforma.

Este planteamiento supondría explicitar, utilizando la expresión de Frabboni (2001), los puntos más y los puntos riesgo, esto es, las potencialidades, las limitaciones y problemáticas relacionadas con los diferentes elementos que configuran los diferentes marcos ecológico-educativos que nos condicionan a todas las personas: el escolar, el familiar y el sociocomunitario.

\section{- El contexto sentido o la mirada vivenciada}

Finalmente, la tercera mirada que nosotros contemplamos como posible supondría dar una vuelta de tuerca más a nuestro análisis contextual, integrando 
lo reflexionado, lo racionalizado, en narrativas vitales o experienciales que le otorguen sentido (esto es, que lo den profundidad), le inyecten fuerza o energía (propiciando su necesario dinamismo) y que integren en él diferentes tipos de elementos: cognitivo-lingüísticos, sociales, afectivos, éticos... (que permitan la construcción de configuraciones holísticas o globales).

Hechos particulares, experiencias concretas, metáforas interiorizadas por una comunidad educativo-escolar se pueden convertir en potentes herramientas interpretativas de la realidad contextual en la que se inscribe nuestra práctica pedagógica.

Desde las anteriores consideraciones sobre la inexcusable tarea del enmarque contextual del Proyecto, creemos que sería necesario plantearse y derivar las siguientes consideraciones para la práctica educativa:

- La superación de las miradas epidérmicas referidas pasa, a nuestro entender, por constatar a partir de actividades sencillas como, por ejemplo, el estudio de casos, concepciones y/o situaciones educativas reales con la habitual fisonomía polisémica que ellas encierran. Sólo a partir de análisis "finos", esto es, matizados de cada una de ellas es desde donde cada persona o, en su caso, sector de la comunidad educativa escolar, puede constatar, valorar y, si éticamente es aceptable, respetar, las perspectivas o miradas de los otros en relación con la situación sometida a análisis.

- Las miradas vivenciadas que requerirían "bucear" en las narrativas de los diferentes "nativos" de esa comunidad para construir una narrativa común y compartida desde un contexto sentido se nos antojan como una meta a alcanzar a largo plazo, una vez superadas otras fases anteriores ya que, el pretenderla alcanzar de forma no reposada y pensada, ha llevado a más de un centro a caer en la frustración y el desencanto.

\subsection{Apuntando algunos problemas, tareas y compromisos}

No queremos finalizar esta primera parte de nuestra reflexión sin apuntar, aunque sea de forma sintética, algunas potenciales limitaciones que nos podemos encontrar en relación con el proceso de construcción y de desarrollo práxico del Proyecto, así como unas sucintas consideraciones acerca de las tareas y retos a asumir en relación con él, además de las ya desgranadas en los anteriores epígrafes.

En relación con las limitaciones, Feito (2006) nos señala dos problemas no resueltos o que no deja claros la LOE. El primero hace referencia a la forma de dar solución al hecho de que un profesor actúe de espaldas o en contra de ese proyecto democráticamente elaborado cuando éste tiene que ser respetado por toda la comunidad educativa. Por su parte, el segundo que contempla se refiere a la necesidad $-\mathrm{y}$ dificultad- de conseguir plantillas docentes estables en los centros que permitan su desarrollo y consolidación. 
Otras potenciales dificultades que podemos considerar serían el entender la identidad que nos confiere el PEC como estado y no como proceso (Essomba, 2006), el no entender la identidad como un proceso de construcción de sentido (Castells, 1997) o el configurar proyectos que no respondan a las ideas fundamentales de sencillez, periodo breve de realización y de no partir de cero sino de la experiencia acumulada en el centro (Beltrán y San Martín, 2000).

Por lo que respecta a las tareas y compromisos que derivarían de nuestro Proyecto señalaríamos, en primer lugar, la necesidad de esforzarnos por y ayudarnos a percibir la importancia que tiene su construcción en cuanto herramienta simbólica que otorga sustrato y direccionalidad a nuestra actividad educativa.

En segundo término, y no menos importante, comportaría entender el Proyecto como texto que nos otorga macrorreferentes explícitos y consensuados, esto es, concebirlo como algo acuñado en virtud de la transición que se produce desde la cultura del yo a la cultura del nosotros.

En definitiva, el principal reto sería hacer nuestro el Proyecto, vivirlo como algo que, al igual que todo lo pedagógico, es dinámico y perennemente inacabado y también soñarlo porque, sin una cierta dosis de utopía, difícilmente se producirán conquistas importantes en educación.

\section{DelosREFERENTES CONSENSUADOSDECENTROALOSVECTORESREGULADORESDELOSPROCESOS EDUCATIVOS DE AULA}

Por muy importante y determinante que sea la asunción por parte de una determinada comunidad educativa de una serie de referentes filosófico-educativos que otorguen valor, sentido y coherencia a la propuesta pedagógica que deseen auspiciar, la concreción última de aquéllos se inscribe en las prácticas de aula en las que cada uno de los educadores desarrolla su tarea educativa.

Y es en cada uno de esos escenarios concretos, con actores (docentes y alumnos) particulares, en donde se configuran y cristalizan o, por el contrario, se empequeñecen o se hacen añicos, los grandes referentes sustentadores acuñados desde el consenso comunitario. Es por ello por lo que es importante considerar la voz de todos aquellos que respiran la cotidianidad pedagógica.

Desde la asunción convencida de que "los procesos que acontecen en el sujeto que aprende para dar significado a la información dependen de las relaciones pedagógicas existentes" (Romero, Bernal y Jiménez, 2009, 13), en esta segunda parte de nuestra exposición someteremos a consideración la relevancia pedagógica que pueden tener un conjunto de postulados y de vectores reguladores de la acción educativa que, más que ser nuevos, se ubican y mueven habitualmente en la periferia pedagógica de nuestra práctica profesional, siendo objeto de frecuentes miradas recelosas o, en el mejor de los casos, ostentando un protagonismo escaso. 


\subsection{Los referentes éticos como soportes fundamentales de la práctica pedagógica}

Aunque -de forma no siempre explícita ni generalizada- podamos seguir constatando en nuestros contextos educativos la presencia de ciertas filosofías y planteamientos pedagógicos reduccionistas-mecanicistas, parece fuera de toda duda que la práctica pedagógica va mucho más allá de la mera dimensión técnica que, obviamente, también ha de contemplar.

En la profesión educadora, como afirman Martínez y Tey (2007), la ética profesional ostenta un valor consustancial, siendo un componente y no un añadido de la misma. Si no fuera así y sólo contempláramos los elementos técnicos, la profesionalidad del profesorado disminuiría ostensiblemente.

En este mismo sentido se expresa Tardif cuando afirma que la dimensión ética no es un elemento periférico de profesiones y ocupaciones que, como la de la enseñanza, están muy vinculadas con las relaciones humanas. Para él, esta dimensión ética se proyecta, se manifiesta en diferentes ámbitos, uno de los cuales está relacionado con las actitudes éticas que los docentes mantienen en relación con los alumnos, con los saberes y con el aprendizaje. En este sentido, afirma que "no se puede negar que tales actitudes, basadas en representaciones, desempeñan un papel fundamental en el aprendizaje. Ciertos maestros hablan excluyendo a los alumnos de su discurso, mientras que otros abren su discurso, dando puntos de apoyo para que éstos puedan progresar" (Tardif, 2004, 108).

En este sentido, nos parece sumamente interesante el trabajo de Meirieu (2001) en el que se nos alerta del peligro que comportaría el que el ámbito técnico se convirtiera en el referente central de la práctica educativa. Así, en muchas ocasiones, la denominada por él fascinación por la herramienta pretende otorgar estabilidad metodológica y honorabilidad científica a un determinado modelo pedagógico, pero con el olvido imperdonable de los otros dos polos que, junto con el praxeológico (en el que se ubicaría aquélla), han de estar siempre presentes en cualquier modelo pedagógico, otorgándole coherencia y consistencia: el de las finalidades, al que también denomina axiológico, y el del apuntalamiento psicológico.

En síntesis, podríamos decir con este profesor francés que toda herramienta pedagógica debe ser puesta en tela de juicio desde el punto de vista ético porque, para él, a diferencia de las disciplinas "científicas" en las que el objeto está mudo o, cuando habla, es porque el científico le hace hablar, en las disciplinas de "acción humana" como es el caso de la pedagogía, el objeto ha de tomar la palabra, porque el objeto es un sujeto y éste, en definitiva, es el que tiene que determinar la pertinencia de lo que se propone en el intercambio comunicativo de la acción pedagógica.

Por su parte, Fullan y Hargreaves (1997) se manifiestan en este mismo sentido cuando afirman que la enseñanza es algo complejo y no reductible a un conjunto de destrezas técnicas, de forma que, más que un oficio técnico, es un quehacer moral.

Y desde esta concepción de la tarea pedagógica como algo consustancialmente moral es desde donde David Hansen (2002) acuña el término de sensibilidad moral para subrayar la importancia de mirar no sólo a lo que un educador 
dice o hace sino, también, a lo que piensa y a cómo actúa. Así, para este autor, términos como confianza, atención, apoyo e implicación están saturados de significado moral. Todos ellos sirven de constatación de la presencia moral del docente en las vidas de los alumnos, aunque, de hecho, aquél casi nunca piense en estos términos ni utilice el término "moral".

La sensibilidad moral, como acertadamente apunta el profesor de la Universidad de Illinois, comporta involucrarse en la tarea en la que todo docente está inmerso. Partiría de la premisa de que la forma en la que los educadores nos aproximamos a las situaciones docentes no es una cuestión de segundo orden sino, más bien, algo determinante. Así, esa sensibilidad moral, integradora de razón y emoción, incluiría la habilidad del docente para retirarse de la escena en determinados momentos para, de este modo, poder reflexionar sobre lo que en ella está ocurriendo y para valorar, entre otras muchas cuestiones, los diferentes puntos de vista que pueden estar presentes en la misma. Esta sensibilidad, como se constata en algunas investigaciones (Jackson, Boostrom y Hansen, 1993), es reconocida por niños y adolescentes en sus profesores.

Por su parte, Goodlad destaca, como uno de los cuatro imperativos morales de la profesión docente, el de construir una relación efectiva profesor-alumno, afirmando que "la responsabilidad moral de los educadores se hace especialmente evidente en el momento en que se cruzan las vidas de los profesores con las de los alumnos" (Goodlad, Soder y Sirotnik, 1990, 49; cit. Fullan, 2002, 22). Por el contrario se constata que, cuando los docentes disminuyen la tonalidad emocional en sus aulas, se suele deber a la escasa consideración que otorgan a todo aquello que vaya más allá de su conocimiento en un ámbito del saber (Hargreaves, 2000).

Concienciarnos de la importancia que ostentan estos referentes morales en el marco de la tarea educativa y asumir los compromisos profesionales que se derivan de ello exigiría que cada docente hubiese determinado, construido, su filosofía de la acción (Núñez Cubero, 2008), imprescindible en cuanto que es la que otorga sentido a su acción pedagógica cotidiana.

Algunas derivaciones prácticas que se pueden desprender de lo dicho podrían ser las siguientes:

- Hemos de ayudar a los docentes, desde diferentes instancias y dinámicas formativas (asesorías externas, seminarios y grupos de trabajo dentro de los centros...) a visibilizar los componentes éticos de sus prácticas y a diferenciarlos de los del plano técnico que también ellas requieren. ¿Qué puede haber de ético y de técnico en la relación que mantengo con mis alumnos?, ¿y en la mantenida con mis compañeros y con las familias? y, ¿en el ámbito de la evaluación?... Visibilizando lo ético a partir de situaciones cotidianas ayudaremos a los docentes a entender y a disfrutar más su práctica, a la vez que a agudizar el compromiso que han de tener con la coherencia requerida en toda actividad educativa de calidad. 
- Sólo logrando que los docentes capten que los modelos pedagógicos (compuestos por diferentes dimensiones o polos) son necesarios para desarrollar prácticas consistentes, estaremos en condiciones de "exigirles" que activen la sensibilidad moral requerida en toda acción pedagógica que se precie.

\subsection{El replanteamiento de la relación pedagógica como tarea inexcusable para mejorar la consistencia, el sentido y el valor del proceso educativo}

Partiendo de la asunción convencida de que toda relación educativa es clave para el desarrollo de una adecuada tarea profesional, será preciso clarificar la fisonomía que habrá de presentar dicha relación para que sea valiosa.

Para nosotros, un requisito fundamental para conseguir este propósito sería garantizar la existencia de lo que podríamos llamar simetría relacional, no entendida ésta en su sentido estricto o absoluto sino, más bien, como elemento favorecedor de las máximas condiciones posibles de simetría con nuestros alumnos dentro de la asimetría institucional y madurativa en la que nos movemos (Gimeno Lorente, 2009). Supondría, en definitiva, valorar positivamente al alumno como interlocutor y sujeto capaz de actuar razonablemente.

Pero, ese tipo de relación deseable se transforma rápidamente en desencanto cuando observamos lo que acontece en muchas de nuestras aulas y escuelas. En este sentido, hemos de ser conscientes de "la tangencialidad o el escaso protagonismo de la mirada y la voz del alumnado en la narrativa pedagógica de las escuelas" (Argos, 2008, 56).

Si entendemos la realidad como algo generado desde la suma total de los aspectos que ofrece un objeto a una multiplicidad de espectadores (Arendt, 1988), si queremos que cada alumno se convierta en actor y sujeto mediante la relación de prácticas y valores (Touraine, 2000), si compartimos la idea de que "aprender es construir mundos donde todos tengan sitio, mundos donde quepan otros mundos, campos semánticos que tengan interfases con otros campos de sentido" (Assmann, 2002a, 106), necesitaríamos acceder a las diferentes perspectivas sostenidas por cada uno de los actores de la escena pedagógica (fundamentalmente docentes y alumnos) para, desde un marco etnográfico-interpretativo, explicitar la perspectiva emic, esto es, la de los "nativos", huyendo de las miradas de aquellos "antropólogos" (léanse ahora docentes) que, bien por inexperiencia, ingenuidad, irresponsabilidad y/o dogmatismo, colonizan los campos semánticos de los restantes actores que comparten con él ese escenario.

Se trataría de conseguir, desde la cultura de cooperación sostenida por Gather Thurler $(2004,74)$, "reducir, a través del intercambio, las diferencias entre las representaciones entre actores y, por tanto, la incertidumbre de la acción pedagógica".

Como apuntan Bransford y otros $(2005$, 86), los conceptos y teorías que mantengamos sobre el aprendizaje son fundamentales en cualquier intento de mejora educativa. Así, todos, incluidos los docentes, construyen asunciones "sobre lo que 
es importante aprender, quién puede aprenderlo, cómo ayudar a aprender y cómo valorar lo aprendido". Por ello, es importante que expliciten sus teorías tácitas, evaluando permanentemente sus asunciones de cara a mejorar a lo largo de su carrera profesional.

Desde la consideración de la dimensión ética como referente nuclear de la práctica educativa y desde el replanteamiento obligado del tipo de relación pedagógica que acabamos de plantear surgen una serie de vectores práxicos que, a modo de ideas o líneas de actuación, hacen que la propuesta educativa por la que abogamos se despliegue con el sentido, la consistencia, la profundidad, la coherencia y el compromiso requeridos.

2.2.1. La pedagogía de la escucha como imperativo ético y axioma generador de un adecuado clima relacional

Este primer vector tiene mucho que ver con el rol de hermeneuta que todo educador ha de desempeñar en cualquier contexto pedagógico. La capacidad para interpretar textos, entre ellos el propio Proyecto Educativo, debe ocupar un lugar central en el desarrollo de su competencia profesional. Y, para ello, se hace totalmente necesaria la captación de la perspectiva emic, personal, particular de cada uno de nuestros alumnos y, en consecuencia, el respeto hacia cada uno de ellos en cuanto actores individuales que sostienen peculiares e idiosincráticas miradas acerca del contexto educativo en el que están inmersos.

Esta pedagogía de la escucha es reconocida como una necesidad, como un requisito para el desarrollo de un clima relacional de calidad en cualquier contexto y nivel educativo y guarda una estrecha relación con la pedagogía de la mirada que plantean Jover y Reyero (2000), que perfilan Bárcena y Jover (2005) y, más concretamente, con el tacto y la capacidad de escucha en cuanto que dimensión que atiende al mundo afectivo y sentimental de los demás.

Reconocer, considerar y escuchar la voz del otro se nos antoja como un imperativo ético y como un referente de primer orden en la tarea pedagógica. Una voz que se proyecta en múltiples lenguajes y que sólo podrá ser adecuadamente interpretada si estamos predispuestos y somos sensibles a ello. Una voz que se teje con hilos no sólo cognitivos sino, también, afectivo-emocionales. Una voz molar, holística, preñada de significado experiencial siempre que se enmarque en un contexto de respeto, confianza y optimismo pedagógico.

Desarrollar comprometidamente esa pedagogía de la escucha comporta generar ambientes pedagógicos de libertad indagadora para que, de ese modo, se puedan desplegar en cada uno de los actores sus capacidades autónomas para aprender y crecer en todos los ámbitos de desarrollo. Ello, como nos apunta Finkel (2008), nos ha de permitir enseñar con la boca cerrada, dejando que sea la indagación la que nos enseñe y la que nos induzca a aprender.

En definitiva, se trataría, como afirma Azerêdo Ríos (2003), de entender como prioritaria la exigencia ética del reconocimiento del otro, prestar atención a la palabra 
de los alumnos, ayudarles en orden a fundamentar esa palabra y a liberarla de las imposiciones restrictivas de su significado.

2.2.2. El aprendizaje lento como elemento favorecedor de la autonomía y de los ritmos de aprendizaje de los alumnos

La modernidad líquida presenta, para autores como Bauman (2007), una serie de características que comportan consecuencias y plantean retos en el ámbito educativo. Y uno de los rasgos definitorios de esta sociedad es lo que el profesor polaco denomina síndrome de la impaciencia, entendido como una forma de ver el tiempo -y su paso- como un fastidio, como una contrariedad; en definitiva, como un ladrón que nos posterga la obtención de recompensas pretendidas.

Tomando en consideración lo anterior, y pensando ahora en clave pedagógica, no es excesivamente difícil percibir el potencial -y constatable- antagonismo existente entre ese contexto de impaciencia y la pedagogía de la escucha a la que acabamos de hacer referencia y que, como hemos visto, plantea la necesidad de captar las diferentes voces que se despliegan en los contextos educativo-escolares. Así, aparecen "ruidos" que, por desgracia, están excesivamente presentes en las relaciones educativas.

La gran mayoría de esos "ruidos" o elementos disonantes no tienen específicamente su origen en el microcontexto escolar sino que están determinados por condicionantes macrocontextuales. Uno de estos condicionantes es el relacionado con las relaciones "bajo presión" que se constatan en una sociedad "hiperexigente" (Romero, Bernal y Jiménez, 2009). Efectivamente, como afirma Romero (2000, 61), "la temporalidad inmediata adquiere su máxima expresión en un universo social regido precisamente por la inmediatez y la velocidad [...]. La urgencia por anticipar el presente dentro del propio presente, hacen que el futuro inmediato introduzca una suerte de violencia en nuestro propio presente". El síndrome de la impaciencia emerge; la impaciencia de la modernidad se encarna también en una suerte de impaciencia pedagógica ininteligible tanto desde el plano moral de la educación como desde la valoración de las metas alcanzadas.

Y esta impaciencia que se visibiliza y constata tanto en alumnos como en educadores nos debería llevar a una reconsideración de nuestros tiempos de enseñanza y de aprendizaje porque, sólo de este modo, creemos que podríamos convertir ese sentimiento de agobio y de presión por el trabajo, en otro que nos permita disfrutar de las provocaciones, los retos y las exigencias que se plantean en el proceso de construcción del conocimiento y en el de desarrollo de todos los actores de la escena pedagógica como personas autónomas y libres.

Y es desde la persecución de este propósito desde donde creemos que puede sernos de utilidad un concepto, una expresión que, en los contextos escolares, ha sido percibida habitualmente como antitética de lo que podría entenderse como deseable: el aprendizaje lento. Éste, acuñado por Guy Claxton en su obra Cerebro de liebre, mente de tortuga, plantea que ciertas formas de acción de carácter 
paciente son las más adecuadas para captar el sentido de situaciones complejas y poco definidas como las que muchas veces caracterizan las relaciones humanas.

Para este profesor, uno de los fines que la educación debería perseguir sería el cultivar un estado mental relajado que permitiera que las cosas "vengan por sí solas". Así habría que superar la obsesión por los cambios apresurados (algo característico del cerebro de liebre) e intentar trabajar desde una mente de tortuga que supondría ser capaces de asimilar y aprender desde situaciones de trastorno y cultivar la capacidad de esperar y de permanecer atentos ante la incomprensión generada por ellas.

Esta pretensión de conseguir conocimiento poco a poco (Fullan, 2002b) contrasta un tanto con la evidencia bastante generalizable de que el tiempo es un problema perenne para los docentes (Hargreaves, Moore y Fink, 2003). Ante esta disonancia, los educadores deberíamos hacer un esfuerzo para no dejarnos llevar por la premura de los logros tangibles, para distanciarnos un tanto de la obsesión por la conquista de aprendizajes a corto plazo, para profundizar junto con nuestros alumnos en análisis multiangulados de las variadas situaciones que se plantean en el curso de la tarea pedagógica.

2.2.3. Rescatando de la trastienda pedagógica algunas actitudes y capacidades valiosas: la revitalización de la intuición, la creatividad, la curiosidad y el placer por aprender

Desde los marcos que generan la pedagogía de la escucha y el aprendizaje lento será posible andamiar otra serie de elementos favorecedores de una educación que pretenda ser algo más que la transmisión mecánica y apresurada de un conocimiento empaquetado.

Estos elementos tal vez podrían entenderse mejor si consideramos el análisis postmoderno que efectúa Kincheloe (2001), en el que aboga por un cambio de los planteamientos de la modernidad y de sus escuelas, de cara a superar su tendencia clara hacia el absolutismo y la certeza, así como su énfasis por lo cuantitativo, lo distante y lo inmediato en detrimento de la calidad, las relaciones y el contexto.

La escuela de hoy, para nosotros, necesita perder el miedo ante lo nuevo, ante todo aquello que "rompe" y reta lo que, de incuestionable y cierto, se respira en ocasiones en escuelas, aulas, libros de texto y también en docentes y estudiantes arrogantes y/o ingenuos. Porque, como acertadamente afirma el argentino Horacio Belgich (2007), cuando el aprendizaje remite a modos mecanicistas, memorísticos y repetitivos, existe una permanente cerrazón de la subjetividad ante lo que considera peligroso, esto es, lo nuevo que invade al sujeto.

Desde las anteriores argumentaciones, se nos antoja imprescindible la aparición en la escena pedagógica de nuevos planteamientos educativos, en los que la curiosidad, la creatividad y el placer están presentes porque, como afirma Assmann 
(2005), aprender con curiosidad conlleva el placer por conocer, comprender, descubrir, construir y reconstruir el conocimiento.

Finalmente, el concepto de intuición y el del conocimiento intuitivo que de ella se desprende aparecen como ingredientes pedagógicos que han sido recurrentemente infravalorados en los contextos educativo-escolares.

La escasa consideración de este concepto que está estrechamente vinculado al del conocimiento lento anteriormente referido suele estar motivada por la distancia (en ocasiones, incluso oposición) que mantiene respecto de los enfoques excesivamente lineales y mecanicistas que suelen presidir las tareas pedagógicas de nuestras escuelas.

En el interesante trabajo editado por Atkinson y Claxton (2002), se nos presentan las potencialidades que tiene este elemento que, para el segundo de estos autores (2002), nos remite a una familia dispersa de "maneras de conocer" menos articuladas y explícitas que el discurso y el razonamiento habituales y que, con frecuencia, ha sido ignorada, marginada y denigrada por las culturas educativas oficiales.

Estos peculiares "modos de saber" característicos de la intuición, aun teniendo relación e incluyendo el conocimiento, comprenden también otros muy importantes: sentimientos, corazonadas y maneras de reconocer esquemas complejos (Furlong, 2002), ostentando un marcado componente experiencial. Serían, en definitiva, "interpretaciones holísticas de situaciones basadas en analogías que surgen de nuestra base de datos inconsciente, fruto de nuestra experiencia" y que integran una gran cantidad de información (Claxton, 2002, 72).

Ahora bien, el afirmar que este modo de conocer sea relevante no nos ha de llevar a inferir que las hipótesis que desde él se generan sean infalibles. Es desde esta constatación desde donde cobra sentido el planteamiento de los profesores Brown y Coles $(2002,222)$ quienes sostienen la importancia de educar la intuición en el ámbito educativo para poder desarrollar, de este modo, algo más que simples "interpretaciones condensadas" y conseguir intuiciones que sean flexibles y capaces de adaptarse a diferentes situaciones y circunstancias.

Estas formas de conocimiento intuitivo las percibimos como bastante afines a la forma que tiene Assmann (2005) de concebir la dinámica del pensamiento que, equiparada por él al "hipertexto virtual", mantiene una estrecha relación con conceptos como curiosidad, creatividad, experiencia y placer de aprender.

De las reflexiones planteadas en relación con los anteriores vectores práxicos, podríamos plantear las siguientes orientaciones o pautas para la acción pedagógica:

- Parece importante abordar de forma conjunta desde los centros una reflexión profunda sobre la pertinencia, delimitación conceptual y formas de concreción práctica de la referida simetría relacional. En relación con ello, nuestra experiencia constata que muchas de las disfunciones que se generan en relación con climas de aula inadecuados o con problemas de disciplina individuales, se podrían haber mitigado si, previamente, hubiera habido una reflexión y explicitación entre docentes, familias y 
alumnos, del marco relacional que se entiende como adecuado en el contexto educativo escolar.

- Sería también fundamental, si es que asumimos que la pedagogía de la escucha debería presidir la tarea pedagógica, considerar los diferentes modos en que ella puede instaurarse. Así, habría que considerar las variadas fisonomías que puede adoptar (mediante el gesto, la respuesta, el silencio, la pregunta, la provocación...) así como sus diferentes niveles de exigencia, tanto en función del tipo de alumno, de la situación o contexto específico, o de cualquier otro elemento condicionante.

- Evitar el síndrome de la impaciencia debería convertirse en otro de los retos de las prácticas pedagógicas. Los tempos en educación son importantes y las prisas muy malas consejeras. Es por ello por lo que los centros en su conjunto y los docentes en particular deberían concienciarse y asumir consensuadamente el valor de la reflexión pausada, del aprendizaje lento, tanto referidos a los alumnos como a nuestra tarea como docentes.

- Remover esquemas, repensar y reconstruir situaciones, mejorar dinámicas relacionales o efectuar cualquier tipo de mejora en la tarea educativa pasa, como hemos visto, por revitalizar la intuición, la creatividad, la curiosidad y el placer por aprender. Ahora bien, el reto práctico debería ir encaminado a entenderlos como elementos fundamentalmente actitudinales que han de estar incorporados en el quehacer pedagógico cotidiano del centro y de las aulas.

Tendríamos que tratar de evitar los frecuentes "desenfoques" que se constatan en docentes y comunidades escolares, generados por entenderlos como "herramientas" o como métodos (técnicas y actividades para el desarrollo de la creatividad y /o la intuición, tiempos de ludismo creativo, planteamientos reduccionistas como los del tipo de aprender jugando...) y no como componentes que impregnan el conjunto de la actividad educativa.

\section{A MODO DE SÍNTESIS}

El planteamiento de fondo que sustenta nuestra reflexión se inscribe, básicamente, en la necesaria dialéctica que, en el ámbito educativo, ha de existir entre los marcos filosófico-educativos compartidos y las asunciones pedagógicas personales de los educadores.

Si bien es cierto que, más que nunca en educación, se hace preciso transitar desde la cultura del yo a la cultura del nosotros, no es menos obvio que ambos planos (el personal-idiosincrático y el socio-grupal) deben convertirse en puntos de referencia obligados que, permitiendo multiangular la reflexión sobre nuestros contextos educativos, hagan más profunda, coherente y fundamentada nuestra tarea pedagógica. 
Todos los referentes textuales, tanto los que son fruto de asunciones consensuadas formalizadas en un determinado proyecto a compartir, como los acuñados desde las coordenadas personales insertas en la filosofía y en las creencias pedagógicas de cada educador, son elementos fundamentales en cuanto que otorgan consistencia al quehacer pedagógico cotidiano. Así, sustentados ambos en referentes axiológicos y teleológicos, se convierten en macrorreferentes de toda filosofía y planteamiento pedagógicos.

Entendemos que de poco o nada serviría el asumir consensos a nivel de centro si, posteriormente, éstos no se proyectan en las prácticas pedagógicas de sus docentes y que, igualmente, poco sentido y valor tendría la existencia de unas coordenadas o de una filosofía de la acción altamente definida por parte de un docente si sólo fuera fruto de un esfuerzo individual tan loable como, a menudo, inservible en cuanto a su escasa repercusión en las dinámicas educativas del centro.

Desde nuestro punto de vista, para que una práctica educativa sea valiosa, necesitaríamos conjugar textos y contextos, tanto grupales como personales; tanto objetivos como mentales. La articulación, la armonización de todos ellos nos permitirá desarrollar miradas multifocales a nuestros principios y prácticas pedagógicas. Ninguno de ellos sobra, la dialéctica de ida y vuelta ha de estar siempre presente. El crecimiento de un docente como tal requiere, habitualmente, ir aparejado con un crecimiento de la comunidad educativa en la que desarrolla su tarea.

El conjunto de reflexiones, postulados y vectores que a lo largo del presente trabajo hemos ido desgranando requerirían, entre otras cosas, el que los educadores ejerciéramos el liderazgo persuasivo al que se refieren Stoll y Fink (1999) cuando se plantean la mejora de nuestras escuelas y que, a su entender, debe sustentarse en el optimismo, el respeto, la confianza y el apoyo intencional como premisas básicas.

Desde premisas de este tipo creemos que sería posible soñar, idear y encarnar pedagógicamente una meta pedagógica tan quimérica como apasionante: la conquista del placer por aprender y, por supuesto también, por educar. Tal vez de este modo, como apunta Assmann (2002), se pueda visibilizar en los educadores el goce de estar contribuyendo a integrar los procesos vitales y el conocimiento.

\section{REFERENCIAS BIBLIOGRÁFICAS}

Angulo, J. F. (2002) La planificación de la mejora de la escuela, en Murillo, F. J. y MuñozREPISO, M. (coords.) La mejora de la escuela. Un cambio de mirada. Barcelona, MEC/ Octaedro, 127-164.

ARENDT, A. (1988) La condición humana. Barcelona, Paidós.

ARgos, J. (2008) Procesos de subjetivación y construcción de la ciudadanía desde el contexto escolar, en Marrero, J. y ARgos, J. (coords.) Educación, convivencia y ciudadanía en la cultura global. Bilbao, Wolters Kluwer, 47-71.

Assmann, H. (2002) Placer y ternura en la educación. Hacia una sociedad aprendiente. Madrid, Narcea. 
- (2005) Curiosidad y placer de aprender: el papel de la curiosidad en el aprendizaje creativo. Madrid, PPC.

Atkinson, T. y ClaXton, G. (eds.) (2002) El profesor intuitivo. Barcelona, Octaedro.

AzERÊDO Ríos, T. (2003) Comprender y enseñar. Por una docencia de la mejor calidad. Barcelona, Graó.

Bárcena, F. y Jover, G. (2005) La ciudadanía imposible. Pensar al sujeto cívico desde una pedagogía de la mirada, en OraIsón, M. (coord.) Globalización, ciudadanía y educación. Barcelona, Octaedro/OEI, 49-82.

Bárcena, F. y Mélich, J. C. (2000) La educación como acontecimiento ético. Barcelona, Cuadernos de Pedagogía/Paidós.

Bauman, Z. (2007) Los Retos de la educación en la modernidad líquida. Barcelona, Gedisa.

Belgich, H. (2007) Autonomía, orden escolar y prevención para la igualdad y la libertad, en Boggino, N. (comp.) Convivir, aprender y enseñar en el aula. Sevilla, Homo Sapiens/ Eduforma, 45-64.

Beltrán, F. y San Martín, A. (2000) Diseñar la coherencia escolar. Madrid, Morata.

Bolívar, A. (1998) Educar en valores. Una educación de la ciudadanía. Sevilla, Consejería de Educación y Ciencia Junta de Andalucía.

BRANSFord, J. y otros (2005) Theories of learning and their roles in teaching, en DarLINGHammond, L. y Bransford, J. (eds.) Preparing teachers for a changing world. San Francisco, Jossey-Bass, 40-87.

Brown, L. y Coles, A. (2002) La toma de decisiones complejas en el aula: el profesor como profesional intuitivo, en Atkinson, T. y Claxton, G. (eds.) El profesor intuitivo. Barcelona, Octaedro, 212-232.

Castells, M. (1997) La era de la información: economia, sociedad y cultura. Madrid, Alianza.

Claxton, G. (1999) Cerebro de liebre, mente de tortuga: Por qué aumenta nuestra inteligencia cuando pensamos menos. Barcelona, Urano.

- (2002) Anatomía de la Intuición, en Atkinson, T. y Claxton, G. (eds.) El profesor intuitivo. Barcelona, Octaedro, 50-75.

Essomba, M. A. (2006) Liderar escuelas interculturales e inclusivas. Barcelona, Graó.

Esteban, F. y Buxarráis, M. R. (2004) El aprendizaje ético y la formación universitaria: más allá de la casualidad. Teoría de la Educación. Revista Interuniversitaria, 16, 91-108.

FeITo, R. (2006) Otra escuela es posible. Madrid, Siglo XXI.

Finkel, D. (2008) Dar clase con la boca cerrada. Valencia, Servicio de Publicaciones de la Universitat de Valencia.

Frabboni, F. (2001) El libro de la pedagogía y la didáctica. Vol. 1: La Educación. Madrid, Ed. Popular.

Fullan, M. (2002a) Las fuerzas del cambio. Explorando las profundidades de la reforma educativa. Madrid, Akal.

- (2002b) Liderar en una cultura del cambio. Barcelona, Octaedro.

Fullan, M. y Hargreaves, A. (1997) ¿Hay algo por lo que merezca la pena luchar en la escuela? Sevilla, Publicaciones MCEP.

FurLONG, J. (2002) La intuición y la crisis de la profesionalidad entre los docentes, en ATKINson, T. y Claxton, G. (eds.) El profesor intuitivo. Barcelona, Octaedro, 29-49.

García Gómez, R. J. (2006) Innovación, cultura y poder en las instituciones educativas. Madrid, MEC/CIDE.

Gather Thurler, M. (2004) Innovar en el seno de la institución escolar. Barcelona, Graó. 
Gimeno Lorente, P. (2009) Didáctica crítica y comunicación. Madrid, Mec/Octaedro.

Goodlad, J.; Soder, R. y SirotniK, K. A. (eds.) (1990) The moral dimensions of teaching. San Francisco, Jossey-Bass.

Hansen, D. T. (2002) Explorando el corazón moral de la enseñanza. Barcelona, Idea Books.

Hargreaves, A. (2000) Mixed emotions: teachers' perceptions of their interactions with students. Teaching and Teacher Education, 16, 811-826.

Hargreaves, A.; Moore, S. y Fink, D. (2003) Enseñar a pesar de la sociedad del conocimiento II: la pérdida de la integridad, en Hargreaves, A. Enseñar en la sociedad del conocimiento. Barcelona, Octaedro, 115-146.

Illeras, J. M. y Medina, A. (2006) La organización deliberativa de las instituciones educativas democráticas, en Ayuste, A. (coord.) Educación, ciudadanía y democracia. Barcelona, Octaedro, 103-129.

Jackson, P. W.; Boostrom, R. E. y Hansen, D. T. (1993) The moral life of schools. San Francisco, Jossey-Bass.

Jover, G. y Reyero, D. (2000) Images of the other in childhood: researching the limits of cultural diversity in education from the stand-point of new anthropological methodologies. Encounters on Education/Encuentros sobre Educación/Recontes sur l'Éducation, 1, 125-152.

Kincheloe, J. L. (2001) Hacia una revisión crítica del pensamiento docente. Barcelona, Octaedro.

Martínez, M. y Tey, A. (2007) El profesorado: profesión y compromiso ético, en Pérez, A. I. y otros Profesorado y otros profesionales de la educación. Barcelona, MEC/Octaedro, 37-76.

MeIRIEu, Ph. (2001) La opción de educar: ética y pedagogía. Barcelona, Octaedro.

NúÑEz CuBERo, L. (2008) Opciones fundamentales para pensar la educación, en NúÑ̃zZ Cubero, L. y Romero Pérez, C. Pensar la educación: conceptos y opciones fundamenta-

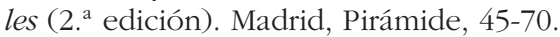

Puig, J. M. y Martín, X. (1998) La educación moral en la escuela. Teoría y práctica. Barcelona, Edebé.

Puig, J. M. y otros (2000) Cómo fomentar la participación en la escuela. Barcelona, Graó.

Romero Pérez, C. (2000) El conocimiento del tiempo educativo. Barcelona, Laertes.

Romero Pérez, C.; Bernal Guerrero, A. y Jiménez Vicioso, J. R. (2009) Tejiendo Vínculos: la textura de la relación educativa, en Peña Calvo, J. V. y Fernández García, C. M. (coords.) La escuela en crisis. Oviedo, Octaedro/Serv. Public. Universidad de Oviedo, 105-149.

Stoll, L. y Fink, D. (1999) Para cambiar nuestras escuelas: reunir la eficacia y la mejora. Barcelona, Octaedro.

TARDIF, M. (2004) Los saberes del docente y su desarrollo profesional. Madrid, Narcea.

Touraine, A. (2000) Igualdad y diversidad. Las nuevas tareas de la democracia (2. ${ }^{a}$ edición). México DF, Fondo de Cultura Económica. 